\title{
APPENDIX 2: MOLECULAR DATA BASES
}

\author{
A few starting points
}

\author{
E.F. VAN DISHOECK \\ Sterrewacht Leiden P.O.-Box 9513 \\ 2300 RA Leiden, The Netherlands
}

\section{Introduction}

Astrochemistry makes enormous demands on laboratory and theoretical chemistry to obtain line frequencies, molecular parameters and rate coefficients for chemical reactions under interstellar conditions. The astrophysical needs for such large data bases continue to increase. For example, spectral line surveys at submillimeter wavelengths routinely contain thousands of rotational lines, whereas high resolution infrared and optical data require large amounts of information on vibrational and electronic transitions. The astrophysical modeling, both of interstellar clouds and of the envelopes and photospheres of stars, has improved to the stage that highly accurate molecular data are essential.

As a result, many compilations of various kinds of molecular data are scattered throughout the chemical and (astro-)physical literature, including this volume. A brief summary is given here. No attempt is made at completeness, but a few starting points are given to facilitate further searches. A significant development in the last few years has been the enormous expansion and availability of such data bases in electronic form (CD-ROM, ftp, World-Wide-Web). Millions of molecular lines ranging from the millimeter to the extreme ultraviolet part of the spectrum can now be accessed online, and improved software allows rapid searches through the data bases. Care should be taken, however, in using such data bases, since not all entries may have been critically evaluated. Users should therefore be aware of the sometimes limited reliability of such data bases, and are urged to check the original literature whenever appropriate. 


\subsection{GENERAL SPECTROSCOPY}

The classic Molecular Spectra and Molecular Structure books by Herzberg $(1945,1950,1966)$ and Huber \& Herzberg (1979) are still a valuable source of information. Bernath (1996) has recently announced a bibliographic data base on spectroscopy of diatomic molecules, which extends the coverage of the literature from 1973-1996 that was previously critically reviewed by Huber \& Herzberg.

The bi-monthly Berkeley Newsletter also continues to be a useful starting point. This Newsletter is essentially a bibliography of articles in the current journals on molecules comprising four or fewer atoms (contact jphillips@astro.berkeley.edu or spdavis@physics.berkeley.edu).

The books Atomic and Molecular Data for Space Astronomy, ed. P.L. Smith \& W.L. Wiese (1992), and Laboratory and Astronomical High Resolution Spectra, eds. A.J. Sauval et al. (1995), contain several chapters by experts, and are useful sources of information. An excellent summary of available molecular spectroscopy data bases is given in $\S 3$ of the chapter by Jørgensen in this volume, including information on the SCAN data base, the Kurucz line lists, and other specific line lists for individual molecules. Finally, CCP7 (http://ccp7.arm.ac.uk/ccp7/) maintains several data bases and programs of relevance for molecular astrophysics.

\subsection{MICROWAVE SPECTROSCOPY}

The classic textbooks are by Townes \& Schawlow (1955) and Gordy \& Cook (1984). Poynter \& Pickett (1984) and Lovas (1992) have provided detailed lists of millimeter transitions of astrophysically important molecules, which continue to be updated and are available on the WWW. They are being expanded to higher frequencies, but are still far from complete in the $\mathrm{THz}$ range.

\subsection{INFRARED SPECTROSCOPY}

The HITRAN data base (Rothman et al. 1992) is a very useful tool for infrared spectroscopy, although it contains only a limited set of molecules, mostly of atmospheric interest. An updated version has been prepared by Chance et al. (1994) and a new 1996 version on CD-ROM containing the new HAWKS (HITRAN Atmospheric Workstation) is available (Rothman et al. 1997). This compilation contains also UV cross sections and parameters, the band-model code MODTRAN3, documentation, and references to critical line-by-line parameters. The GEISA data base is described by Husson et al. (1992). 
Additional information for astrophysically relevant molecules can be found in Crovisier (1992). See also Jørgensen, this volume. A high resolution infrared spectrum of Arcturus for astronomical reference has been published by Hinkle et al. (1996).

\subsection{OPTICAL AND UV SPECTROSCOPY}

Morton (1991) summarizes the resonance line data for astrophysically important atoms, and Morton \& Noreau (1994) review the electronic spectrum of the CO molecule. See Smith \& Wiese (1992) and the CFA WWW site for related atomic and molecular data for astronomy.

\subsection{REACTION RATE COEFFICIENTS}

Chemical models of interstellar clouds typically contain hundreds to thousands of reactions, including ion-molecule, neutral-neutral, dissociative recombination, radiative association, photoionization and photodissociation processes. Most of the available information has been included in the UMIST data base (Millar et al. 1997) and continues to be updated. Information and programs are available through CCP7.

A recent compilation of ion-molecule reactions has been published by Anicich (1993). Laboratory data of neutral-neutral reactions are available from NIST, and are updated on an annual basis (Mallard et al. 1994).

\subsection{COLLISIONAL EXCITATION}

Flower (1990) summarizes in his Appendix 2 the available collisional excitation rate coefficients at that time, most of which stem from theoretical calculations by the late Sheldon Green and co-workers. Green's collection of collision data for astrophysically interesting molecules is still being maintained in electronic form http://molscat.giss.nasa.gov/Data/MCRates/.

\section{References}

Anicich, V.G. 1993, J. Phys. Chem. Ref. Data 22, 1469; ApJS 84, 215

Bernath, P.F. 1996, private communication (bernath@watsci.uwaterloo.ca (demo version available through anonymous $\mathrm{ftp}$ on watsci.uwaterloo.ca in /pub/DiRef)

Chance, K., Jucks, K.W., Johnson, D.G., Traub, W.A., 1994, J. Quant. Spectrosc. Rad. Transf., 52, 447; http://firs-www.harvard.edu/

Crovisier, J., 1992, Constants for molecules of astrophysical interest in the gas-phase: photodissociation, microwave and infrared spectra, Obs. de Meudon

Flower, D. 1990, Molecular Collisions in the Interstellar Medium (Cambridge University Press)

Gordy, W., Cook, R.L. 1984, Microwave Molecular Spectra, 3rd edition (Wiley, New York) Herzberg, G. 1945, Molecular Spectra and Molecular Structure. II. Infrared and Raman Spectra of Polyatomic Molecules, (Van Nostrand Reinhold, New York) 
Herzberg, G. 1950, Molecular Spectra and Molecular Structure. I. Spectra of Diatomic Molecules, (Van Nostrand Reinhold, New York)

Herzberg, G. 1966, Molecular Spectra and Molecular Structure. III. Electronic Spectra and Electronic Structure of Polyatomic Molecules, (Van Nostrand Reinhold, New York)

Huber, K.P., Herzberg, G. 1979, Molecular Spectra and Molecular Structure. IV. Constants of Diatomic Molecules, (Van Nostrand Reinhold, New York)

Husson, N., Bonnet, B., Scott, N.A., Chedin, A. 1992, J. Quant. Spectrosc. Rad. Transfer 48,509

Hinkle, K., Wallace, L., Livingston, W., 1995, Infrared atlas of the Arcturus spectrum 0.9-5.3 $\mu \mathrm{m}$ (ASP, San Francisco); available by anonymous ftp to 140.252.1.11 in /pub/hires_k_band and /pub/lowres_k_band

Jørgensen, U.G. 1997, this volume.

Lovas, F.J., 1992, J. Phys. Chem. Ref. Data, 21, 181; http: //physics.nist.gov/PhysRefData/

Mallard, W.G., Westley, F., Herron, J.T., Hampson, R.F., Frizell, D.H. 1994, NIST Chemical Kinetics Database: Ver. 6.0, National Institute of Standards and Technology, Gaithersburg, MD; http://www.nist.gov/srd/

Millar, T.J., Farquhar, P.R.A., Willacy, K. 1997, A\&AS in press; http: //saturn.phy.umist.ac.uk/

Morton, D.C. 1991, ApJS 77, 119

Morton, D.C., Noreau, L. 1994, ApJS 95, 301

Poynter, R.L., Pickett, H., 1985, Appl. Optics, 24, 2335; http://spec.jpl.nasa.gov/

Rothman, L.S., et al., 1992, J. Quant. Spectrosc. Rad. Transfer, 48, 469; available on CD-ROM; http://www.HITRAN . com, LRothman@CfA. Harvard.edu

Rothman, L.S., et al., 1997, J. Quant. Spectrosc. Rad. Transfer, in press; available on CD-ROM; http://www. HITRAN.com, LRothman@CfA.Harvard.edu

Sauval, A.J., Blomme, R., Grevesse, N., eds., 1995, Laboratory and Astronomical High Resolution Spectra, ASP Conference Series Vol. 81 (Astronomical Society of the Pacific, San Francisco)

Smith, P.L., Wiese, W.L., eds., 1992, Atomic and Molecular Data for Space Astronomy (Springer, Berlin), 149; http: //cfa-www.harvard.edu/amp/data/

Townes, C.H., Schawlow, A.L. 1955, Microwave Spectroscopy, (Dover, New York) 